\title{
Human Genome Variation
}

\section{We wholeheartedly endorse this new journal concentrating on variants causing and associated with disease and trait phenotypes, especially the Data Report format for rapid publication and the database dissemination of the full spectrum of findings from a single familial variant to a comprehensive locus review.}

$\mathrm{T}$ he Japan Society of Human Genetics and Nature Publishing Group have launched Human Genome Variation (http://www.nature.com/hgv/), a sister journal to the Journal of Human Genetics, with the intention of accelerating the annotation of the human variome. Current journals are making progress in this area, but we acknowledge that focusing on the excellence and impact of the work does not by itself guarantee that the results of the research are accessible to those who need them in a timely way and in a form they can use. Peer review improves your paper. It also improves the journal when the editors use referee comments to set benchmarks and criteria. So the publishable unit becomes larger, review becomes more exacting and the knowledge turn becomes longer, inhibiting further research and clinical translation. It is not surprising that the standards of proof for variants in hereditary monogenic diseases are high and remarkably similar among journals, given the large number of journals competing for articles (164 in this year's Thomson Reuters Genetics and Heredity category alone) and sharing a pool of referees.

We emphasize that we are selecting articles that offer a high standard of genetic evidence (rather than surrogate molecular or cellular function), research or translational tools for a large specialist user group. In particular, we and our referees favor those research reports with 'key' discoveries that provide the first insight into a new genetic mechanism, disease causation or biological principle (Nat. Genet. 44, 1, 2012). On page 1239 of this issue, Kristijan Ramadan and colleagues describe mutations in SPRTN, encoding a DNA damage sensor, that result in early-onset hepatocellular carcinoma and an associated premature aging syndrome. On page 1245, Gregor Andelfinger and colleagues report a novel role for the cohesin complex, in which SGOL1 mutations affect the pacemakers of both cardiac and smooth muscle rhythms.
Several journals have found publication formats that balance the need to provide articles with sufficient content to attract readers and citation with the duty to help identify and database all the variants in the human genome that contribute to disease phenotypes and traits, including Human Mutation's Mutation Updates and our own community annotation reviews-most recently, of Lynch syndrome and cystic fibrosis variants (Nat. Genet. 46, 93, 2014).

In this issue, we offer a large community reassessment of malaria risk variants (see page 1197). In this article, the MalariaGEN Consortium confirms classic associations with the severe malaria subphenotypes of cerebral malaria and severe malarial anemia. These include sickle cell trait $(H B B$, heterozygous protective rs334), blood group $\mathrm{O}(A B O$, recessive protective rs8176719), ATP2B4 (rs10900585 conferring dominant risk) and CD40LG (recessive protective rs3092945). Rather surprisingly given its extensive literature, G6PD deficiency (rs1050828) is currently nearly neutral overall with respect to severe Plasmodium falciparum malaria owing to opposite effects on the two subphenotypes, leading the authors to suggest that numerous textbooks need to be rewritten to reevaluate the evidence for G6PD deficiency as an ancient Plasmodium vivax resistance mechanism in Africa before the Duffy antigen sweep.

We commend Human Genome Variation, as few journals have had the courage to rapidly report single disease-associated variants with only minimal genetic evidence (single de novo or familial incidence). Indeed, we went so far as to suggest that such reports should be rapidly published only as protected preprints (Nat. Genet. 45, 1411, 2013). It remains to be seen whether peerreviewed Data Reports provide the best way to identify valid variants in genetic disorders and move them into the databases or whether there is still a need to tag variants of interest even earlier in the chain of evidence, as we have proposed. 